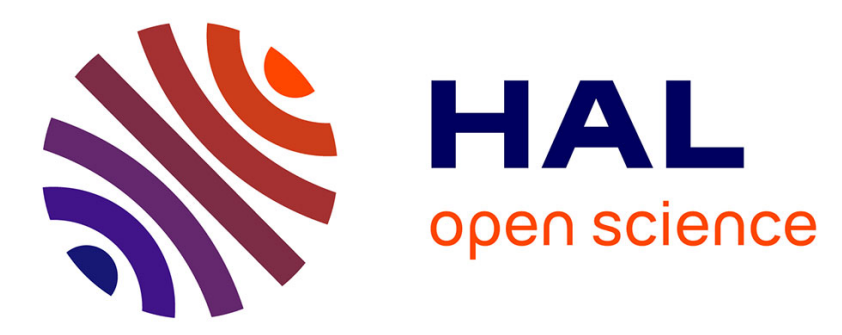

\title{
Challenges obtaining a biowaiver for topical veterinary dosage forms
}

Ronald E Baynes, Jim Riviere, Thomas Franz, Nancy A Monteiro-Riviere, Paul Lehman, Mathieu Peyrou, Pierre-Louis Toutain

\section{- To cite this version:}

Ronald E Baynes, Jim Riviere, Thomas Franz, Nancy A Monteiro-Riviere, Paul Lehman, et al.. Challenges obtaining a biowaiver for topical veterinary dosage forms. Journal of Veterinary Pharmacology and Therapeutics, 2012, 35 (1), pp.103-114. 10.1111/j.1365-2885.2012.01381.x . hal-01191257

\section{HAL Id: hal-01191257 \\ https://hal.science/hal-01191257}

Submitted on 1 Sep 2015

HAL is a multi-disciplinary open access archive for the deposit and dissemination of scientific research documents, whether they are published or not. The documents may come from teaching and research institutions in France or abroad, or from public or private research centers.
L'archive ouverte pluridisciplinaire HAL, est destinée au dépôt et à la diffusion de documents scientifiques de niveau recherche, publiés ou non, émanant des établissements d'enseignement et de recherche français ou étrangers, des laboratoires publics ou privés. 


\section{Challenges obtaining a biowaiver for topical veterinary dosage forms}

\author{
R. BAYNES* \\ J. RIVIERE* \\ T. FRANZ ${ }^{\dagger}$ \\ N. MONTEIRO-RIVIERE* \\ P. LEHMAN ${ }^{\dagger}$ \\ M. PEYROU ${ }^{\ddagger} \&$ \\ P.-L. TOUTAIN ${ }^{\S}$ \\ *College of Veterinary Medicine, North \\ Carolina State University, Raleigh, NC, \\ USA; ${ }^{\dagger}$ Cetero Research Corp., Fargo, ND, \\ USA; ${ }^{\star}$ Novartis Animal Health, Basel, \\ Switzerland; ${ }^{\S}$ Ecole Nationale Veterinaire de \\ Toulouse, Toulouse, France
}

\author{
Baynes, R., Riviere, J., Franz, T., Monteiro-Riviere, N., Lehman, P., Peyrou, M., \\ Toutain, P.-L. Challenges obtaining a biowaiver for topical veterinary dosage \\ forms. J. vet. Pharmacol. Therap. 35 (Suppl. 1), 103-114.
}

Obtaining a biowaiver for topical drugs used in veterinary species faces many of the same challenges associated with human topicals. However, the skin of domestic animals varies anatomically and biochemically and experimental approaches to assess bioequivalence (BE) in veterinary species have challenges that are not often encountered with human skin. This is especially the situation with locally acting drugs. The focus of this paper is to address several of the challenges associated with (i) determining the BE of these locally acting drugs and (ii) critically examine the current technological advances that can act as a surrogate for clinical trials.

(Paper received 18 December 2011; accepted for publication 30 December 2011)

Ronald E. Baynes, College of Veterinary Medicine, North Carolina State University, 1060 William Moore Dr., Raleigh, NC 27607,USA.E-mail:ronald_baynes@ncsu.edu

\section{BACKGROUND OF THE PROBLEM}

Veterinary topicals constitute a large segment of the veterinary pharmaceutical market. Several of these products that are approved by the US FDA are deemed to be systemically absorbed and require a veterinary prescription and/or consultation based on potential systemic and/or adverse effects in the animal. Topically applied antiparasitic drugs constitute the majority of these drugs that are systemically absorbed. Most of the remaining US FDA-approved veterinary topical are antibiotics, corticosteroids, and NSAIDs that work locally, and systemic absorption is not expected to be 'significant' for many of these US FDA-approved drugs. Table 1 provides three examples of related US FDA- and EMEA-approved drugs whose systemic disposition is required for adequate efficacy claims. Finally, the majority of drugs topically applied to the skin of veterinary species are US EPA-registered drugs (Table 1) and chemicals intended to act locally, and significant dermal absorption is not anticipated with these topical formulations. The vast majority of these products sold in the United States and EU do not require a prescription and sold over-the-counter. The focus of this white paper is to address several of the challenges associated with (i) determining the bioequivalence (BE) of these locally acting drugs and (ii) critically examine the current technological advances that can act as a surrogate for clinical trials. For formulations expected to have a systemic effect, international harmonization efforts have resulted in accepted conventional PK approach (i.e., demonstration of equivalence of plasma AUC, $C_{\max }$ ), and therefore will not be addressed in this paper. Comparative in vivo clinical trials are challenging as they are difficult to perform especially in veterinary species, costly, highly variable, and consequently often insensitive to formulation differences.

For topical formulation intended to develop only a local action (but not on the skin itself) as ectoparasiticides, EMEA provided recently some information on how to obtain marketing authorization of a generic ectoparasiticidal spot-on product for dogs and cats. (EMEA/CVMP (2009). It indicated that the efficacy of a proposed product should be confirmed usually in two controlled clinical studies per parasite species on the target animal species, using the least susceptible species (i.e., for fleas = Ct. felis (the almost only and most prevalent on dogs and cats); for ticks: least susceptible tick species). The selection of the at least susceptible tick species should be justified, based on in vitro studies with the determination of LD50 and LD90 or on appropriate literature, if available. It can be argued that a new product developed using this approach is not a true generic (no comparative trial, no statistics); in addition and as for all topical formulation, interaction between formulation (vehicle) and many environmental factors (e.g., rain) should be expected (PL Toutain, personal communication).

The FDA has provided several guidance documents in the last 12 years for determining the BE of human topical drugs. It is clear from these documents that the development of scientifically sound surrogate methods for clinical trial endpoints has been challenging and therefore will be even more challenging for veterinary species. The current FDA guidance for industry as outlined by in 'Topical Dermatological Corticosteroids: In vivo Bioequivalence' (FDA, 1995) is limited to corticosteroids and can only be applicable to human skin and not to the skin of veterinary species. Two physically similar corticosteroid 


\begin{tabular}{|c|c|c|c|}
\hline $\begin{array}{l}\text { Dermal } \\
\text { disposition }\end{array}$ & Trade name & Active ingredients & Species approval \\
\hline $\begin{array}{l}\text { Systemic/ } \\
\text { FDA drugs }\end{array}$ & $\begin{array}{l}\text { Revolution } \\
\text { Surpass } \\
\text { Ivomec } \\
\text { Synalar Cream Veterinary }\end{array}$ & $\begin{array}{l}\text { Selamectin } \\
\text { Diclofenac } \\
\text { Ivermectin } \\
\text { Flucinolone acetonide }\end{array}$ & $\begin{array}{l}\text { Cats and dogs only } \\
\text { Horses only } \\
\text { Beef cattle only } \\
\text { Dogs only }\end{array}$ \\
\hline $\begin{array}{l}\text { Topical/ } \\
\text { EPA } \\
\text { products }\end{array}$ & $\begin{array}{l}\text { Betagen Topical Spray } \\
\text { K9 Advantix } \\
\text { Frontline Top Spot } \\
\text { Ecto F724 }\end{array}$ & $\begin{array}{l}\text { Betamethasone, Gentamicin } \\
\text { Imidacloprid, Permethrin } \\
\text { Fipronil } \\
\text { Pyriproxyfen }\end{array}$ & $\begin{array}{l}\text { Dogs only } \\
\text { Dogs only } \\
\text { Dogs and cats } \\
\text { Dogs and cats }\end{array}$ \\
\hline
\end{tabular}

Table 1. Examples of veterinary drugs approved for dermal application formulations can be compared by measuring the vasoconstriction response to the corticosteroid formulations. This has often been known as the human skin blanching assay or vasoconstrictor assay (VCA). This approach cannot be used with most veterinary species as the skin in many of these animals is pigmented.

Determining the BE of veterinary topical products faces challenges similar to or greater than those challenges associated with human topical products. This is especially the case for drugs that are not systemically absorbed from topical application, and BE comparisons cannot be ascertained from blood levels. In these situations, BE could rely solely on in vivo clinical efficacy and safety endpoints, and one can expect substantially greater variability in the dose-response relationship than the doseblood concentration relationship (Martinez et al., 2002). BE studies based only on clinical endpoints will therefore require more subjects than if blood drug concentration comparisons were the basis for the BE assessment. There is also the case for two topical products having significantly different bioavailability, reflecting different blood concentrations, and yet having identical clinical response. The latter is often associated with the initial and terminal portions of a sigmoidal dose-response curve, and CDER has therefore recommended that a clinical endpoint BE study to include an administered dose at a point along the linear portion of the dose-response curve.

The topical dose situation can be analogous to orally administered drugs intended for local action as is often the case with antiparasitic drugs frequently used in veterinary medicine. FDA guidance (FDA, 2003) in those situations for human oral drugs suggest that 'suitably designed and validated in vitro studies, if the latter studies are either reflective of important clinical effects or are more sensitive to changes in product performance compared to a clinical study'. The latter sections of this paper will highlight the strengths and weaknesses of several proposed surrogate methods that may be used in the future to assess BE of veterinary topicals.

\section{FACTORS INFLUENCING DERMAL ABSORPTION}

\section{Comparative skin anatomy and absorption mechanism}

Percutaneous absorption and the rate of absorption must take into consideration that anatomical factors may affect the barrier function of skin. The basic architecture of skin is similar in all mammals; however, differences exist in the thickness of the epidermis and dermis between species and within the same species in various regions of the body (Monteiro-Riviere et al., 1990). Differences that exist in biochemistry, microcirculation, hair follicle density and structure, sebaceous gland density, adnexal appendages, and distribution of epidermal cell types are important considerations. One of the major challenges with veterinary topicals is related to the extreme variability between breeds and within a given breed, between seasons, sex, and skin physiology. The presence of sebaceous gland is under control of sex hormones (possible sex effect including effect of castration, circannual rhythms, etc.) and again interaction formulation. The mechanism of chemical and drug transport occurs from the skin surface across the various epidermal layers, through the basement membrane, and into the capillaries in the superficial dermal papillary layer for a systemic effect. The challenges associated with comparing veterinary topical drugs are that the rate and extent of absorption may vary across and within veterinary species because of anatomical and physiological differences.

Species comparison of skin anatomy and physiology and anatomical factors that affect barrier function are very important variables (Monteiro-Riviere, 1991, 2006, 2008a; MonteiroRiviere et al., 2008b) in assessing topical drug delivery. A comprehensive study comparing the histological thickness and laser Doppler blood flow measurements was performed at five cutaneous sites (buttocks, ear, humeroscapular joint, thoracolumbar junction, and abdomen) in nine species (cat, cow, dog, horse, monkey, mouse, pig, rabbit, rat) to determine the correlation of blood flow and thickness. These studies strongly suggested that LDV blood flow and skin thickness did not correlate across species and body sites but are independent variables that must be evaluated separately in dermatology, pharmacology, and toxicology studies (Monteiro-Riviere et al., 1990).

The role of the cutaneous vasculature was studied in the topical delivery of a nonsteroidal anti-inflammatory drug. Skin penetration of ${ }^{3} \mathrm{H}$ piroxicam gel was evaluated by in vitro diffusion cells and in vivo (pigs) at two different tissue beds, one that is vascularized by direct cutaneous and the other by musculocutaneous arteries. The in vitro fluxes were identical, indicating a similar rate of stratum corneum and epidermal absorption; however, more extensive and deeper tissue penetration was noted at the musculocutaneous sites (Monteiro-Riviere et al., 1993). 
Hair follicle arrangement is different in the domestic species. Single hair follicles are found in horses and cattle and are distributed evenly. Pigs have single follicles grouped in clusters of two to four follicles; clusters of three are most common in young weanling pigs. Compound follicle of dogs consists of a single large primary hair and a group of smaller secondary underhairs with diversity in different dog breeds. German Shepherds tend to have a greater number of secondary hairs. Short-coat breeds like the Rottweiler and terriers have primary hairs. Cats have single large primary hair follicle surrounded by clusters of two to five compound follicles, and each compound follicle has three primary hairs and between six and 12 secondary hairs. Sheep have hair (fleece also referred as fibers)-growing regions with single follicles in the face, the distal part of the limbs, and the ear, while woolgrowing regions have compound follicles over most of the body. Goats have single primary hair follicles in groups of three, and three to six secondary hair follicles are associated with each group.

Breed differences are expected, and therefore does a $\mathrm{BE}$ demonstrated in short hair dog or cat be extended without difficulty to long hair dog or cat? While there have not been adequate studies to clearly demonstrate these breed differences within a species, the dermal absorption literature suggests that because of the larger surface area associated with increased hair density, the follicular route becomes more important for drug penetration (Mills \& Cross, 2006). Furthermore, one can question whether BE can be demonstrated in cattle in winter when the air temperature is low compared to summer conditions when the skin temperature is higher.

Many species have hairless areas, such as in the anal canal, the teat of horses, and the internal layer of the prepuce of some species, sebaceous glands empty directly onto the skin surface. Some species have well-developed accumulations of sebaceous glands, some of which are associated with sweat glands that include the infraorbital, inguinal, and interdigital regions of sheep, the base of the horn of goats, anal sacs of cats, and the prepuce and circumanal region of dogs but some areas such as the foot pads, hoofs, claws, and horn, lack sebaceous glands. Other glands such as apocrine glands produce a viscous secretion and are found throughout most of the skin unlike that of humans where they are distributed in the axillary, pubic, and perianal regions. In horse skin, these glands are responsible for the visible sweat during exercise, and at high temperatures, while in goats and cats, they are the least active.

Several studies have demonstrated lateral movement of the drug following topical application and proposed that this phenomenon could be explained by the migration of the drug in the sebum (Jenkinson et al., 1986; Cochet et al., 1997; Chopade et al., 2010). Using microautoradiography, the latter two studies demonstrated this phenomenon with fipronil and imidacloprid applied to the back of the neck of dogs. The drugs were detected at the lumbar region but no radioactivity in the dermis or hypodermis.

A very good example of species differences was demonstrated for topical selamectin, which is approved in the United States and EU for control of several internal and external parasites in cats and dogs. The significant species differences in $C_{\max }$ and AUC values (Sarasola et al., 2002) suggest differences in skin morphology on selamectin diffusion across dog and cat skin. The same conclusions can be made for transdermal fentanyl patches, which are not officially approved for veterinary use, but widely used to control postsurgical pain in cats and dogs. Compared to dogs, cats have shorter lag time (12 vs. 24 h), reduced steady-state flux and absorption (36\% vs. $64 \%$ dose), and formed more persistent depot in skin similar to humans after patch removal (Kyles et al., 1996; Lee et al., 2000). Species differences have also demonstrated for abamectin absorption across the skin of pigs, sheep, cattle, and goats (Baynes, 2004). The latter was demonstrated in vitro and across three liquid formulations, and it is premature to assume that clinical trials would have detected these species differences.

\section{Formulation effects}

Most experimental studies and risk assessments of chemical dermal absorption reported in the literature use neat chemicals or single vehicles such as water, alcohol, or some other organic solvent. However, most exposures are to more complex mixtures. Numerous studies have demonstrated that vehicles have significant effects on modulating chemical absorption (Baynes \& Riviere, 1998; Riviere et al., 2003, 2010). In fact, this is the prime strategy behind developing pharmaceutical formulations. Today, there is little doubt that vehicle and formulation components modulate dermal absorption or transdermal delivery of topically applied drugs and solutes. Excipients are added in the formulations for various performance endpoints, including increasing absorption, decreasing evaporation, retaining formulation on skin, or in some cases to prevent wash-off from rain. The nature of the excipients also determines the colligative properties of the dosage form (e.g., liquid, creams, ointment, or gel). It is expected that dermal absorption across dosage forms would be significantly different.

Table 2 below lists where interactions that would modulate absorption of an active ingredient could occur in the skin. This determines their mechanism of action. Some vehicle effects are so reproducible that quantitative structure activity relationships have been developed to predict the effect of formulation components on absorption (Riviere \& Brooks, 2005, 2011; Ghafourian et al., 2010). These studies demonstrate that the

Table 2. Mechanisms of potential mixture effects on dermal absorption

Surface of skin: • Chemical-chemical (binding, ion-pair formation, etc.)

- Altered physical-chemical properties (e.g. solubility, volatility, critical micelle concentration) $\bullet$ Altered rates of surface evaporation $\bullet$ Occlusive behavior $\bullet$ Binding or interaction with adnexial structures or their products (e.g. hair, sweat, sebum)

Stratum corneum: • Altered permeability through lipid pathway (e.g.

enhancer) • Altered partitioning into stratum corneum $\bullet$ Extraction of intercellular lipids

Epidermis: • Altered biotransformation • Induction of and/or modulation of inflammatory mediators

Dermis: • Altered vascular function (direct or secondary to mediator release) 
physical-chemical properties of the formulation additive alter active drug absorption. Pure in vitro methods assessing chemical partitioning into a series of membrane-coated fibers have also been used to predict mixture effects on chemical absorption across skin (Riviere et al., 2007; Baynes et al., 2008).

These data suggest that any topical formulation is a unique entity that will have a different extent and rate of absorption, requiring regulatory assessment. Much of the mixture studies to date suggest that some of formulation-induced modulation of drug absorption can be both predicted from inert systems and assessed in vitro. However, some of the interactions are a function of the degree of biological complexity seen in the test system used, making interactions seen with in vitro diffusion cells different than seen with vascularized skin (Riviere \& Brooks, 2011). The most obvious situation where this applies would be with vasoactive ingredients, irritants resulting in vasodilation and dermal inflammation, or metabolic modifiers that alter firstpass dermal metabolism or cell transport activity. Exposure to systemic compounds may even modulate the degree of absorption of a simultaneously applied topical compound (Riviere et al., 2003).

Another caveat occurs when complex mixtures are involved, which have opposite effects on absorption. For example, two formulations of very different compositions could have identical transdermal absorptive fluxes if formulation One contained additive $A$ that bound drug to the surface and additive $B$ that enhanced epidermal permeability; while formulation Two contained additive $C$ that prevented surface binding and additive D that reduced epidermal permeability. Although transdermal flux may be similar between formulations, dermal residence time and skin deposition could be different. This was clearly seen when jet fuel hydrocarbon absorption was assessed across fuels with different performance additive packages (Muhammad et al., 2004). As is true with other routes of administration, BE must be assessed both by rate and extent of absorption, endpoints that would reflect the kinetics of dermal penetration. For topical dosage forms, degree of skin penetration might also be needed to differentiate formulations with excipients with very different mechanisms of action.

\section{Grooming behavior}

Animal behavioral traits such as grooming/licking as evidenced in cattle and cats is a behavioral factor when controlled in clinical trials do not duplicate drug disposition in field conditions. Toutain et al. (2012) describes this often overlooked phenomena in more detail in another paper in this supplement issue. Bioequivalence when licking is prevented therefore does not guarantee BE in field conditions. Herd et al. (1996) first reported the higher fecal concentrations of ivermectin following pour-on than following subcutaneous injection, although there were lower plasma concentrations following the pour-on route. Laffont et al. (2001) and Bousquet-Mélou et al. (2011) later confirmed these pharmacokinetic observations by controlling for the natural grooming behavior. Dupuy et al. (2004) reported PK differences following topical selamectin in dogs and attributed the differences to more pronounced grooming behavior in female dogs compared to male dogs as well as possible greater clearance in males than in females. This grooming behavior may account for significant variability in systemic availability that is unpredictable. Untreated animals in a treated herd show a greater variability than the treated animals, and Bousquet-Mélou et al. (2011) have suggested that this can lead to potential selection window for anthelmintic resistance. While the aforementioned discussion has been focused on the systemically available drugs, there have been no reported studies describing how this grooming behavior influences the dermal disposition and efficacy of veterinary topicals that do not require systemic absorption to be efficacious. It can be assumed that the same issues associated with variability will compound a $\mathrm{BE}$ assessment.

\section{SURROGATE METHODS: IN VITRO}

\section{In vitro release test}

The in vitro rate of release test (IVRT) for semi-solid topical drug products has proven to be a useful manufacturing quality control tool for comparing batch-to-batch performance characteristics by measuring the rate of release of the active pharmaceutical ingredient (API) through a porous membrane into a suitable receptor solution. It is especially valuable when making changes to already approved products (small changes in the components or composition of the formulation, certain changes in manufacturing equipment, process, or site) in that the need to conduct a full BE study to establish the BE of the new and old products can be satisfied by demonstrating the 'sameness' of the two formulations through IVRT (FDA, 1997, SUPAC-SS Guidance). Although the FDA did not suggest its use in establishing the BE of generic topical drug products, the situation faced with generic topicals is remarkably analogous to those described in SUPAC-SS in which certain changes to a product's formulation can be approved using a surrogate test for BE, IVRT. If two products are truly identical, no matter who manufactured them, the rates of release of the API will be identical. Data defining the relative rate(s) of release can be accurately determined by this model and used within an established statistical evaluation to compare test vs. reference formulations.

The model is based on the diffusivity of an active ingredient through the matrix of the vehicle (Higuchi, 1961):

$$
Q=A \sqrt{D \times C s \times\left(2 c_{0}-c_{S}\right)} \times \sqrt{t}
$$

\section{Q, Released amount of API at time $t$ (gm)}

A, Diffusion area $\left(\mathrm{cm}^{2}\right)$

D, Diffusion coefficient $(\mathrm{cm} / \mathrm{min})$

$\mathrm{c}_{\mathrm{S}}$, Saturation solubility of API in the formulation $(\mathrm{gm} / \mathrm{mL})$

$\mathrm{c}_{0}$, API concentration in the matrix $(\mathrm{gm} / \mathrm{mL})$

$t$, time (min)

which results in a linear expression of the cumulative release of the API when plotted to the square root of time. The slope of the line defines the rate of release (Fig. 1). 


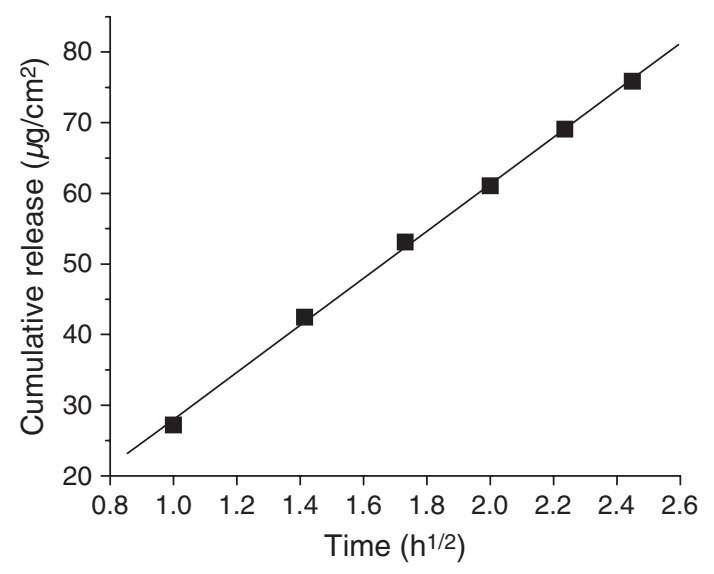

Fig. 1. Graphic representation of cumulative drug release to the square root of time. Solid line is the linear regression of the data.

The applicability of this model was expanded to semi-solid topical formulation evaluations by Shah et al. (1991). The elements of the in vitro IVRT model are quite simple, and this method is in common use for human topical semi-solid drug products. To quote the SUPAC-SS Guidance (FDA, 1997): 'In vitro release method for topical dosage forms is based on an open chamber diffusion cell system such as a Franz cell system, fitted usually with a synthetic membrane. The test product is placed on the upper side of the membrane in the open donor chamber of the diffusion cell and a sampling fluid is placed on the other side of the membrane in a receptor cell. Diffusion of drug from the topical product to and across the membrane is monitored by assay of sequentially collected samples of the receptor fluid'.

Its applicability to veterinarian pharmacology is self-evident as no biological system or tissue is used, just the formulation of interest. The IVRT method also has value in the development of generic formulations, to determine the vehicle matrix equivalence to the reference formulation, before embarking into more involved or expensive BE studies. For example, cream formulations are often very complex mixtures of oils and water and typically require a number of specific manufacturing steps, in which any change in the process may alter the characteristics of the formulation. The IVRT method can be used to demonstrate how well the test vehicle matrix compares to the reference listed drug (RLD) during the development process.

\section{Method development and validation}

Development and validation of the IVRT method to be used follow a logical and sequential process. The same Franz cell used for in vitro skin percutaneous absorption testing can be used for IVRT studies (Fig. 2).

Selection of the reservoir solution and a porous membrane must take into consideration the solubility and stability of the API in the solution, and interaction and binding potential to the membrane. The appropriateness of the reservoir solution and membrane is evident in the subsequent validation of the method. Validation must show day-to-day reproducibility (Fig. 3), ability

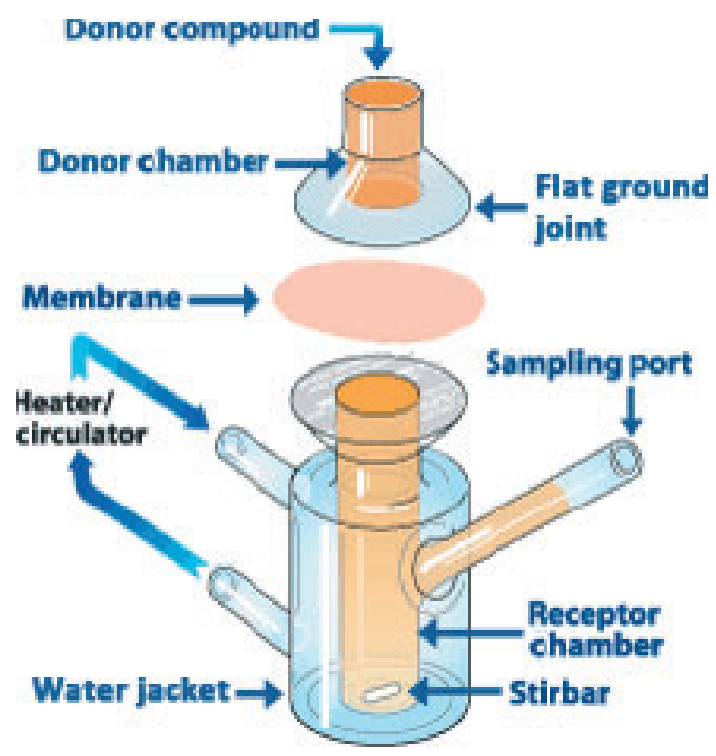

Fig. 2. Schematic representation of a Franz Diffusion Cell used for in vitro rate of release test studies (diagram courtesy of PermeGear, Inc. Hellertown, PA 18055).

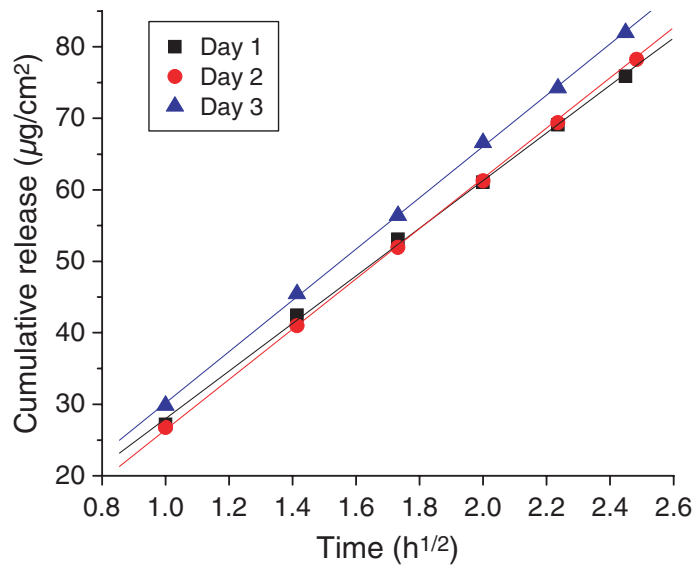

Fig. 3. Example of in vitro rate of release test method reproducibility. Method tested on three different days ( $n=6$ chambers/day), using the reference formulation, showing equality across days based on the SUPAC-SS equivalence criteria.

to discriminate different API concentrations (sensitivity) (Fig. 4), and the ability to differentiate intentionally altered formulations (selectivity) (Fig. 5). Further, if the data are to be submitted as part of a regulatory filing, a fully validated analytical method should be used (ICH Harmonised Tripartite Guideline, 2005).

\section{Case study example}

An example for the value of the IVRT method became evident, many years ago, when a pharmaceutical company was developing a generic ointment steroid formulation. The vehicle was simply USP grade white petrolatum with API. However, when the clinical efficacy endpoint study failed to demonstrate BE to the reference formulation, an investigation was conducted. 


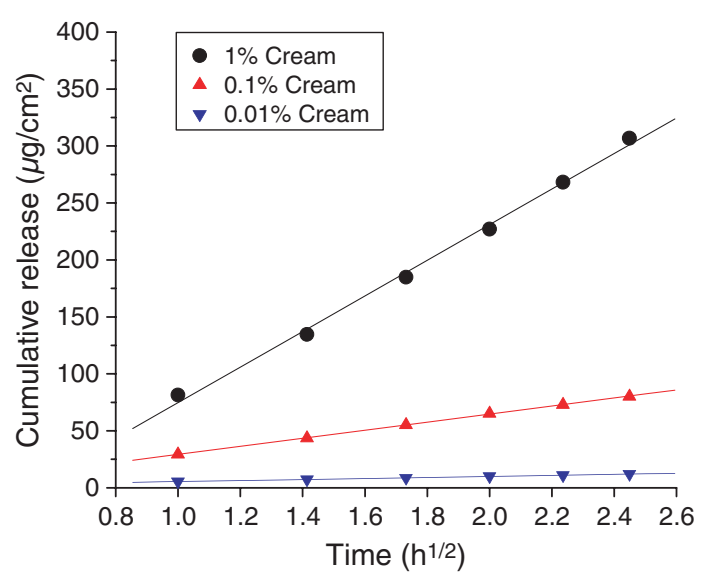

Fig. 4. Example of in vitro rate of release test method sensitivity based on different concentrations of active pharmaceutical ingredient in vehicle ( $n=6 /$ concentration), vs. the reference formulation, showing nonequality across formulations based on the SUPAC-SS equivalence criteria.

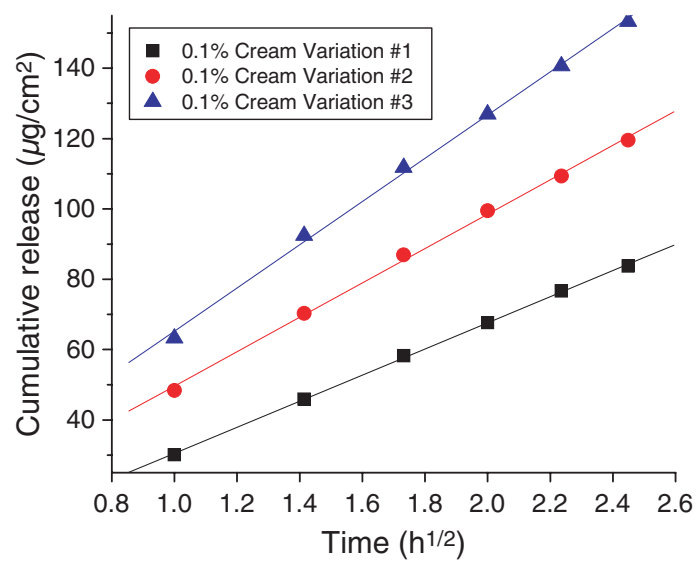

Fig. 5. Example of in vitro rate of release test method selectivity based on intentionally altered vehicles containing active pharmaceutical ingredient ( $n=6 /$ formulation), vs. the reference formulation, showing nonequality across formulations based on the SUPAC-SS equivalence criteria.

Given that the vehicle was only petrolatum, and that USP definition for petrolatum is relatively nonspecific (because of the nature of the excipient), it was determined that the manufacturing source and grade of petrolatum should be the target for investigation. An IVRT method was developed, and six different manufacturing providers of petrolatum were evaluated with the API, and compared to the marketed reference formulation. The summary results of the IVRT comparison are shown in Fig. 6. First, one can see that different manufacturing sources of petrolatum did produce different rates of release for this particular steroid, possibly related to the differences in the distribution ratios of the chain-lengths of hydrocarbons. The original failing generic formulation had used the petrolatum from Source 6 . The data indicated that petrolatum from Source 2 would be a better match to the reference. Subsequently, the use of the Source 2 petrolatum confirmed equivalency in both in vitro skin percutaneous absorption tests and did demonstrate BE in a clinical end-point study. Had this test been performed in early

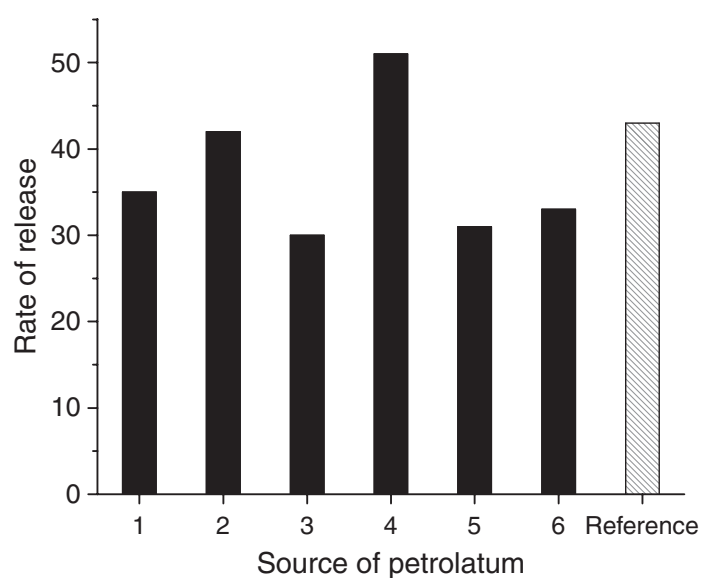

Fig. 6. Case study example on influence of manufacturing source of petrolatum had on the rate of release of a topical steroid.

product development, at the time when the petrolatum source was being selected, the costly failure of the clinical trial could have been avoided.

The in vitro rate of release test for semi-solid topical drug products has proven to be a valuable manufacturing quality control tool for comparing batch-to-batch performance characteristics, as a function of the rate(s) of release of the active ingredient(s). It has also found a use for generic formulation development by determining how close the generic formulation matrix behaves in comparison with the reference formulation. Although differences in the rate of release of the API between test and reference formulations may have no therapeutic significance because they do not correlate with bioavailability, the best current evidence suggests that similar rates of release do signify comparable bioavailability when dealing with formulations that are Q1 and Q2 equivalent.

\section{Skin permeation test}

Evaluation by the FDA of the use of in vitro preparations of human skin to measure percutaneous absorption as a potential surrogate in the area of topical bioavailability and BE began in 1986, but a lack of suitable validation data hindered further consideration of the method at that time (Skelley et al., 1987). Presently, the situation is distinctly different and use of this model as part of a program to establish the BE of topical human or veterinary products can be rationally justified (Franz et al., 2009; Lehman et al., 2011). International acceptance of the use of excised skin as a surrogate for the measurement of in vivo percutaneous absorption underscores the fact that there is general recognition of the validity of the model (OECD 2004, WHO 2005). In this country, the EPA accepts in vitro human data for registration and re-registration of pesticides as part of the 'parallelogram' or 'triple-pack' approach to risk assessment (Reifenrath et al., 2011; Ross et al., 2011).

The elements of the in vitro model are quite simple. To quote OECD 28 (OECD 2004), "The test preparation is applied to the surface of excised skin, which is mounted in a diffusion cell. The 
receptor fluid, which must have an adequate capacity to solubilise the test substance, is maintained in contact with the underside of the skin from the time of application until the end of the collection of the receptor fluid. The test preparation remains on the skin for a specified period of time, relating to potential human exposure, and then the test preparation is removed by an appropriate cleansing procedure. The receptor fluid is sampled at time points throughout the experiment to ascertain the mass (and possibly rate) of the test substance (including any significant metabolite) passing through the skin. At the end of the study, the dislodgeable dose, the amount associated with the skin and the amount in the receptor fluid is determined. These data are necessary to calculate the total skin absorption, and allow for an estimate of the total recovery of the test substance.'

Although the emphasis of most in vitro studies has been directed toward questions relating to human pharmacology and toxicology, the fundamentals of the method apply equally to veterinary pharmacology and toxicology. With respect to its potential use in establishing the BE of topical animal products, one critical question is how good is in vitro/in vivo (IVIV) correlation.

\section{In vitro/in vivo correlation}

Several recent publications have presented a compilation of data from many studies conducted over the past $40+$ years that demonstrate the validity of the in vitro human skin model (Franz et al., 2009, Lehman et al., 2011). As discussed below, not only is there excellent quantitative concordance between in vitro and in vivo data when using total absorption as the metric for comparison; but, where tested, the model has also been shown to prospectively predict the $\mathrm{BE}$ of generic formulations prior to pivotal clinical evaluation.

The quantitative agreement between in vitro and in vivo data can be seen in the publication of Lehman et al., 2011;. A systematic review of 92 data sets from 30 published studies demonstrates a clear clustering of the in vitro and in vivo data around the line of a perfect 1:1 correlation (Fig. 7a). The average IVIV ratio for all data sets is 1.6 , showing a tendency for greater absorption in vitro than in vivo. Because of the lack of harmonization between the in vitro and in vivo protocols in many of the cited studies (different skin sites, different vehicles, etc.), variability was large, and for any single compound, the
IVIV ratio could vary from 0.18 - 19.7. A subset analysis of 11 studies in which perfect harmonization between the in vitro and in vivo protocols was documented showed the true relevance of the model (Fig. 7b). In this case, the average IVIV ratio approached one (0.96) and variability was substantially reduced (0.58-1.28). The conclusion is clear, and data obtained from the in vitro model can accurately duplicate that obtained in vivo when all the critical parameters governing absorption in vivo are replicated in the model.

Data demonstrating good quantitative correlation between in vitro and in vivo results have also been found in animal studies. A summary of the data from several major studies can be found in the recent publication of Ross et al. (2011).

\section{Bioequivalence assessment}

The ability of the excised human skin model to accurately quantify the absorption of topically applied compounds strongly supports the hypothesis that it is an ideal model by which to establish the $\mathrm{BE}$ of topical products. Data to support this argument have been supplied by Franz et al. (2009). In a prospective study, multiple generic formulations of each of five glucocorticoid products were compared to multiple lots of each reference product (RLD). On the basis of assessment of relative bioavailability (total absorption/48 h), the test formulation and reference lot that most closely matched were then taken to a pivotal clinical trial (in vivo VCA). In all five cases, the test products were found to be bioequivalent to the reference products and subsequently approved by the FDA with an $\mathrm{AB}$ rating.

Comparison of the in vitro data to the clinical data shows that, in four of five cases, the results were virtually identical and both tests found a test/reference ratio close to one (Table 3). Assessment of relative bioavailability in the model accurately predicted the BE of test and reference product. This is also supported by the examination of the rate of absorption data in which the kinetic profiles of the test product mirrored that of the reference product (Fig. 8). In vitro equivalence was not seen with the fifth glucocorticoid, mometasone furoate, in which the test/reference ratio was 0.63 . However, the reason for this was found to be because of the insensitivity of the clinical assay, not a failure of the in vitro method. One example of the striking lack of sensitivity of the clinical VCA is seen in Table 3, where it can be noted that there is no difference between alclometasone
Fig. 7. Correlation between total absorption (\% of dose) determined both in vitro and in vivo for (a) 92 data sets from 30 published studies plotted on log-log scale, and for (b) 11 fully harmonized data sets plotted on log-log scale. Solid line represents the ideal one-to-one correlation and dashed lines represent \pm three-fold difference from ideal. Data taken from Lehman et al. (2011).
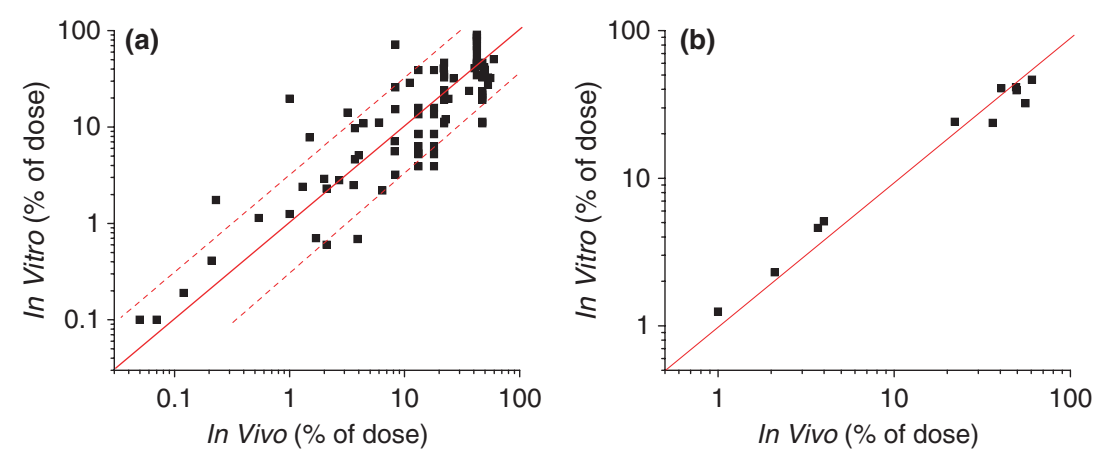
Table 3. In vitro/in vivo comparison of five generic glucocorticoid products (test) vs. the corresponding reference products

\begin{tabular}{|c|c|c|c|c|c|c|}
\hline & \multicolumn{3}{|c|}{$\begin{array}{c}\text { In vitro } \\
\text { absorption*, }{ }^{*} \\
\mathrm{AUC}_{0-48 \mathrm{~h}}\end{array}$} & \multicolumn{3}{|c|}{$\begin{array}{c}\text { In vivo } \\
\text { vasoconstrictor } \\
(\mathrm{VC}) \text { assay* } \\
\text { Negative } \\
\text { AUEC }_{0-24 \mathrm{~h}}\end{array}$} \\
\hline & Test & Ref & $\begin{array}{c}\text { Test/ } \\
\text { ref }\end{array}$ & Test & Ref & $\begin{array}{c}\text { Test/ } \\
\text { ref }\end{array}$ \\
\hline Alclometasone cream & 4.52 & 4.39 & 1.03 & 18.5 & 16.8 & 1.10 \\
\hline Alclometasone oint. & 66.95 & 70.0 & 0.96 & 16.0 & 17.4 & 0.92 \\
\hline Halobetasol cream & 110.4 & $96.9 *$ & 1.14 & 33.1 & 30.7 & 1.08 \\
\hline Halobetasol oint. & 246.7 & 256.3 & 0.96 & 28.6 & 28.5 & 1.00 \\
\hline Mometasone oint. & 213.4 & 338.7 & 0.63 & 13.7 & 12.3 & 1.11 \\
\hline
\end{tabular}

Data taken from Franz et al. (2009).

*Listed numbers are mean values.

${ }^{\dagger} \mathrm{AUC}_{0-48 \mathrm{~h}}=\mathrm{ng} / \mathrm{cm}^{2} / 48 \mathrm{~h}$.

\#Average of three reference lots, none of which were used in the VC study. In all other comparisons, identical lots of test and reference products were used in both the in vivo and in vitro studies.

ointment and cream in the VCA despite a 15-fold difference in absorption.

A second study to demonstrate the utility of the excised skin model in establishing $\mathrm{BE}$ was conducted on two AB-rated generic tretinoin gels, $0.01 \%$ and $0.025 \%$ (Spear Pharmaceuticals) Franz et al. (2009). In contrast to the prior work with glucocorticoids which was performed as a pilot study and not powered to meet FDA statistical standards, the tretinoin study was performed as a simulated BE study in which the goal was to meet the $80-125 \%$ confidence interval requirement. Analogous to the procedure used in oral BE studies, test and reference products were compared with respect to three primary endpoints: total absorption (AUC, $\mathrm{ng} / \mathrm{cm}^{2} / 48 \mathrm{~h}$ ), maximum rate of absorption $\left(J_{\max }, \mathrm{ng} / \mathrm{cm}^{2} / \mathrm{h}\right)$, and time of maximum rate of absorption $\left(T_{\max }, \mathrm{h}\right)$. In agreement with the clinical efficacy data upon which regulatory approval was based, equivalence was also found in the in vitro model (Table 4). The confidence intervals for all three primary endpoints were within the interval 0.80-1.25 for the $0.01 \%$ tretinoin gel, and for two of three endpoints with $0.025 \%$ tretinoin. The third endpoint $\left(J_{\max }\right)$ barely failed at 1.27 .
In summary, although the emphasis of most in vitro percutaneous absorption studies has been directed toward questions relating to human pharmacology and toxicology, the fundamentals of the method apply equally well in the veterinary field as the barrier-controlling absorption in both human and animals of interest is the stratum corneum. The ability of the model to yield results in agreement with clinical data, when comparing test and reference human products for BE, supports its use as part of the regulatory process for the approval of generic topical drug products. The excellent concordance between in vitro and in vivo data with respect to total absorption also supports the position that this test provides an accurate means by which to establish the BE of generic topicals. An obvious advantage to use of the in vitro model vis-à-vis animal generic topicals is the ability to evaluate $\mathrm{BE}$ in each individual animal species approved for given drug product in a cost-efficient and time-efficient manner.

\section{SURROGATE METHODS: IN VIVO}

There are various in vivo tests that have the potential to serve as surrogates for clinical trials in establishing BE. Some of these tests have been or are currently being considered for use in the approval of human generic drug products. An excellent review on the subject that discusses the topic of in vivo surrogate methods in a more thorough manner than will be attempted here is that of Herkenne et al. (2008). It is unfortunate, however, that their utility in the veterinary area may be of limited or no value because of important differences between animal and human skin.

\section{Vasoconstrictor assay}

The only test approved as a surrogate for human generic topicals is the vasoconstrictor (VC) assay, which is used to establish the $\mathrm{BE}$ of topical glucocorticoids. The test utilizes a pharmacodynamic approach based on the ability of glucocorticoids to cause constriction of the superficial dermal vasculature leading to blanching of the skin. The degree of blanching is assessed over a period of $24 \mathrm{~h}$ or more using a chromameter, from which one can calculate an area-under-the-effect-curve (AUEC). As this pharmacologic effect is undoubtedly related to the amount of drug entering the skin, the AUEC is an indirect measure of the
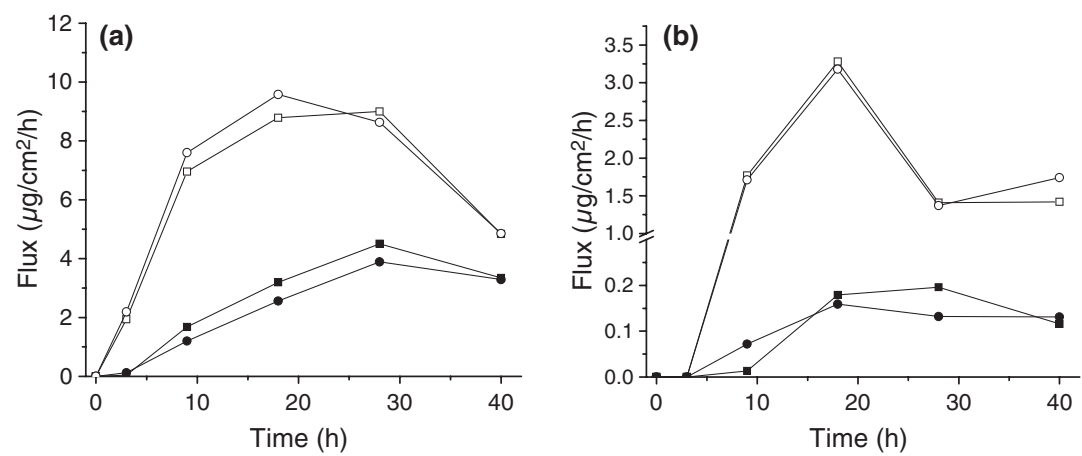

Fig. 8. In vitro percutaneous absorption of (a) Halobetasol propionate, and (b) Alclometasone dipropionate. Test Cream, • Reference Cream; $\square$ Test Ointment, $\bigcirc$ Reference Ointment. Data taken from reference Franz et al. (2009). 
Table 4. Comparison of primary endpoints for test and reference tretinoin gels in the excised human skin model

\begin{tabular}{lcccc}
\hline & Test & Reference & Test/reference & $90 \%$ CI \\
\hline \multicolumn{4}{c}{$0.01 \%$ tretinoin gel } \\
\cline { 2 - 4 } & 2.996 & 2.974 & 1.02 & $97.07,107.46$ \\
$J_{\max }$ & 0.549 & 0.572 & 1.04 & $92.53,115.05$ \\
$T_{\max }$ & 3.596 & 3.573 & 1.04 & $92.23,116.37$ \\
& \multicolumn{4}{c}{$0.025 \%$ tretinoin gel } \\
AUC & 3.492 & 3.471 & 1.03 & $95.14,110.45$ \\
$J_{\max }$ & 0.906 & 0.884 & 1.11 & $95.08,127.88$ \\
$T_{\max }$ & 3.664 & 3.725 & 0.98 & $97.26,99.52$ \\
\hline
\end{tabular}

Statistical analysis was based on natural log-transformed data. The estimated error standard deviation was used to compute the $90 \%$ confidence intervals for the ratios of the means (test/ref) of the listed parameters. Data taken from Franz et al. (2009). AUEC, area-under-theeffect-curve.

drug's bioavailability. Comparison of a test and reference formulation based on the ratio of their AUECs, therefore, becomes a reasonable way by which to establish BE. A Guidance providing detailed instructions on the conduct of the test and analysis of the data has been provided (FDA, 1995).

Several practical issues present a significant barrier to the use of this test in animals. The presence of both fur and heavily pigmented skin would make the test difficult or impossible to apply. Even with shaving or in animals with sparse hair, the problem of pigment would likely reduce the sensitivity of the test. This issue has never been adequately addressed in human studies as the screening procedure excludes subjects who are not 'responders' to a test application of the steroid. Excluded subjects may truly have been those with a poor vasoconstrictor response, or they may have been those with moderate to heavily pigmented skin in whom a good response could not be seen by the detector. This information has never been collected. Another major concern in dealing with animals is that an accurate chromameter reading can only be taken with the subject perfectly still. Although future research may find limited application of this test in certain situations, and on that basis the data could be accepted to establish BE, widespread use of the VC assay is highly improbable.

\section{Stratum corneum tape stripping}

Commonly referred to as the dermatopharmacokinetic (DPK) method, sequential tape stripping of the stratum corneum to obtain drug levels as a function time is a potentially useful BE tool with no regulatory standing at the present time. It was initially proposed as a one-size-fits-all solution to demonstrate the BE of topical generic drug products. An analogy was made between topical drug delivery and oral drug delivery in which both the stratum corneum and systemic circulation represent intermediate compartments through which drugs must travel to reach their site of action. Therefore, just as AUC and other kinetic parameters derived from blood samples can be used to establish the BE of oral drugs, the same paradigm can be followed with topical drugs. The method was considered so promising by the Office of Generic Drugs that a Draft Guidance was issued in 1998. However, in 2002, the Guidance was withdrawn after contradictory results were obtained by two laboratories that were attempting to validate the method. Since that time FDA sponsored research has led to a much better definition of the tape-stripping procedural details and an understanding of the protocol deficiency that led to the earlier validation failure (N'Dri-Stempfer et al., 2009). This group of investigators has also successfully demonstrated the potential use of the DPK method in establishing the BE of topical antifungal drugs. Although the rather limited experience with the tape-stripping method results in there being inadequate data to support its widespread use for all therapeutic classes of generic topical drugs, its use to establish the BE of topical antifungals seems undeniably appropriate as the site of action itself is being sampled.

As with the VCA, several practical issues present a significant barrier to the use of this test in the veterinary area. The dosing and sampling procedures are time-consuming and would require the animals to be still for extended periods of time. Another problem is the heavy density of hair present in many animals. Even after shaving a large proportion of the skin surface would be covered by stubble, which might significantly interfere with sampling of the stratum corneum. The thinness of the stratum corneum in most animals relative to that of human is also of concern and raises the question as to whether the method would be as sensitive as it appears to be in human. The present lack of any relevant studies in which an attempt has been made to evaluate the $\mathrm{BE}$ of topical generics in animals generates moderate uncertainty around the utility of this technique. Currently, it seems plausible that the method could be adapted for use with antifungal drug products.

\section{Microdialysis}

Microdialysis is currently a hot topic in dermatologic research and it is frequently mentioned as a potentially useful technique for establishing the $\mathrm{BE}$ of topical drug products. Although its ability to directly sample interstitial fluid in the dermal compartment is the great attraction of the method, a number of very practical issues present significant roadblocks to its routine use. Herkenne et al., 2008 give an excellent summary of the studies that have been conducted to date and discuss the pros and cons of its possible use to demonstrate BE. Under no circumstance would it be a universal test applicable to all therapeutic classes of topical drugs as those of low permeability and high protein binding are presently too challenging analytically. Water-soluble drugs of moderate permeability appear to be the best candidates for this technique. No simulated BE study to demonstrate the successful use of microdialysis has yet been conducted.

A number of questions can be raised regarding the suitability of this method for BE testing. As there is a limited amount of time $(5-10 \mathrm{~h})$ the dialysis probe can be left in place, this would be inadequate for drugs in which the maximum flux occurs at $>10 \mathrm{~h}$. There is also the need to control the movement of an 
animal during the placement of the dialysis probe and over the many hours during which fluid is being pumped through probe and samples continually collected. Presently, microdialysis seems better suited for drug development purposes, where knowledge of the drug concentration within the skin is critical for assessing its potential for therapeutic effectiveness. Although the routine use of microdialysis for establishing the BE of topical animal products seems unduly burdensome, in principle, it should be a valid method for use with some topical drugs.

\section{Transepidermal water loss (TEWL)}

Measurement of TEWL is a pharmacodynamic assay in which the normal rate of water loss through the skin to the atmosphere is used to noninvasively monitor changes in the function of the stratum corneum barrier. Topically applied drugs such as retinoids and higher concentrations of salicylates (to treat corns/calluses/warts in human) can cause significant increases in TEWL following subchronic use or when applied under occlusion. The utility of the method in demonstrating the bioeqivalence of several retinoid products in human has been successfully shown (Franz et al., 1995). There may be limited use of this technique in the veterinary area because no retinoidcontaining products are available. As with the VCA, animals would need to be still for the measurement of TEWL as the positioning of the probe is critical to getting reproducible readings.

\section{Skin biopsy}

The ability to measure a drug's concentration at its site of action, and show equal delivery of drug from test and reference formulations, is the ideal method by which to establish BE. For topical antifungal drugs, stratum corneum tape stripping can be used as the stratum corneum is the site of action. For all other topical drugs whose intended site of action is within the skin, biopsy of the skin would be the most direct approach to demonstrating the $\mathrm{BE}$ of prospective generic products. Although the specific site of action within the skin may not be known, epidermis vs. dermis, that is of no consequence as the entire skin thickness can be obtained. Analysis of the amount of drug in the skin can be performed in such a way as to define drug content by compartment: (i) unabsorbed drug on the surface of the skin, (ii) drug in epidermis, and (iii) drug in dermis.

As some drug products are applied without first shaving the skin, the amount of drug actually reaching the surface of the skin is a critical factor and could differ between test and reference products if there were significant differences in their composition. A skin biopsy study would be conducted in such a manner that, whether applied to shaved or unshaved skin, the first step would be to shave the skin (if an unshaved application) and conduct a surface wash to determine the amount of drug on the skin surface. The wash procedure would have been previously validated to demonstrate both reproducible and quantitative recovery following a brief (1-min) application; though, with some drugs, quantitative recovery may not be possible. Following the wash, a biopsy would be taken, separated into epidermis and dermis, and each tissue extracted and analyzed separately for drug content. Alternatively, there may be cases in which partitioning of the drug into the skin is very low and analysis of the biopsy tissue as a whole might be considered an adequate procedure. Antimicrobials whose intended action is on the skin surface could fit into this category. An additional method to determine the equality of test and reference drug distribution within the skin would be to section the biopsy on a cryomicrotome.

The great advantage of the skin biopsy as a means of establishing $\mathrm{BE}$, as opposed to other in vivo methods, is the reduced need to restrain the animal to take instrumental reading or continuously collect interstitial fluid samples, all of which are sensitive to movement. Also, in contrast to a pharmacodynamic assay, a skin biopsy is a direct measure of drug content and, in some cases, drug metabolites or degradants.

\section{Spectroscopic techniques}

Confocal Raman and Near Infra-Red (Near IR) spectroscopy are the most advanced noninvasive in vivo methods to assess drug diffusion across the skin (Narkar, 2010). The major advantage of these techniques is that they can provide real-time measurements of drug diffusion with the confocal method being semiquantitative and the NIR method being more quantitative. The major disadvantage is that these techniques require that the drug of interest possesses distinct spectral features that distinguishes it from that of skin spectrum. These techniques have been evaluated in human skin albeit with very few drugs (e.g., econazole, estradiol, retinol) and with in vitro skin systems with NIR methods. There has been no assessment with skin from various veterinary species whose skin spectrum would most likely vary within and across species.

\section{SUMMARY}

There is the necessity to distinguish between formulations intended to cause a systemic action from those intended to act only locally either on or in the skin itself. Regarding the latter, attention should therefore be focused on determining what assessments and surrogate approaches can be used to adequately determine the BE for topical drugs which can be classified predominantly as antimicrobials, anti-inflammatory, antifungals, ectoparasiticides. There is significant variability in dermal absorption across species and even anatomical regions within the individual animal. The grooming behavior, which is unique for the veterinary species and not human, also adds to the variability. These sources of variability are compounded by the formulation effects, which can significantly influence drug release and surface disposition for topical drugs.

In vitro methods have been used successfully with human skin BE studies and can be a useful surrogate for in vivo studies. For topical drugs, systemic absorption parameters will not be the focus as absorption will be limited, but rather deposition into the various epidermal and dermal layers could be compared using 
internationally accepted in vitro diffusion systems to mimic the in vivo topical exposures. Promising in vitro - in vivo correlations with human skin does, however, show some promise as surrogate approaches for systemic absorption and it is reasonable to assume that this approach can be applied to topical drugs and across various veterinary species, although very little of this in vitro - in vivo research for topicals has been reported for human skin. In vivo methods such as tape stripping, which has been an issue in human BE studies, is not applicable to the skin of veterinary species. However, the skin biopsy approach may hold the most promise.

Finally, systemic absorption endpoints are not applicable for drugs that only penetrate and work locally, and there is readily available technology that can be used by sponsors to derive BE endpoints for such topical drugs using a combination of surrogate in vitro and in vivo approaches described earlier. One solution could involve a hierarchical approach with empirical and theoretical components that would adequately answer the question of BE for these drugs.

\section{CONFLICTS OF INTEREST}

R.B. declares no conflicts of interest; J.R. has received grant support from Novartis and Pfizer Animal Health within the past two years; T.F. has not declared any conflicts of interest. N.M.-R. declares no conflicts of interest; P.L. declares no conflicts of interest; M.P. is an employee of Novartis Animal Health.; P.-L.T. declares no conflicts of interest.

\section{REFERENCES}

Baynes, R.E. (2004) In Vitro dermal disposition of Abamectin (Avermectin $\mathrm{B}_{1}$ ) in livestock. Research in Veterinary Science, 76, 235-242.

Baynes, R.E. \& Riviere, J.E. (1998) Influence of inert ingredients in pesticide formulations on dermal absorption of carbaryl. American Journal of Veterinary Research, 59, 168-175.

Baynes, R.E., Xia, X.R., Imram, M. \& Riviere, J.E. (2008) Quantification of chemical mixture interactions that modulate dermal absorption using a multiple membrane coated fiber array. Chemical Research in Toxicology, 21, 591-599.

Bousquet-Mélou, A., Jacquiet, P., Hoste, H., Clément, J., Bergeaud, J.P., Alvinerie, M. \& Toutain, P.L. (2011) Licking behaviour induces partial anthelmintic efficacy of ivermectin pour-on formulation in untreated cattle. International Journal of Parasitology, 41, 563-569.

Chopade, H., Eigenberg, D., Solon, E., Strzemienski, P., Hostetler, J. \& McNamara, T. (2010) Skin distribution of imidacloprid by microautoradiography after topical administration to beagle dogs. Veterinary Therapeutics, 11, E1-E10.

Cochet, P., Birckel, P., Bromet-Petit, M., Bromet, N. \& Weil, A. (1997) Skin distribution of fipronil by microautoradiography following topical administration to the beagle dog. European Journal of Drug Metabolism and Pharmacokinetics., 22, 211-216.

Dupuy, J., Derlon, A.L., Sutra, J.F., Cadiergues, M.C., Franc, M. \& Alvinerie, M. (2004) Pharmacokinetics of selamectin in dogs after topical application. Veterinary Research Communications, 28, 407-413.

EMEA/CVMP (2009) Testing and evaluation of the efficacy of antiparasitic substances for the treatment and prevention of tick and flea infestations in dogs and cats Doc. Ref. EMEA/ CVMP/EWP/82829/2009. European Medicines Agency Veterinary medicines and inspections, London, UK.

FDA (1995) Guidance for Industry: Topical Dermatologic Corticosteroids: In Vivo Bioequivalence. Center for Drug Evaluation and Research, http://www.fda.gov/downloads/Drugs/GuidanceComplianceRegulatory Information/Guidances/ucm070234.pdf (last accessed 4/20/11).

FDA (1997) Guidance for industry. Nonsterile semisolid dosage forms. Scale-up and postapproval changes: chemistry, manufacturing, and controls; in vitro release testing and in vivo bioequivalence documentation. CDER (SUPAC-SS CMC 7)

FDA (2003) Guidance for industry: bioavailability and bioequivalence studies for orally administered drug products - general considerations. US Department of Health and Human Services Food and Drug Administration Center for Drug Evaluation and Research (CDER), March 2003.

Franz, T.J., Lehman, P.A. \& Franz, S.F. (1995) Bioequivalence methodology for tretinoin products. Pharmaceutical Research, 12 (Suppl), S245.

Franz, T.J., Lehman, P.A. \& Raney, S.G. (2009) Use of excised human skin to assess the bioequivalence of topical products. Skin Pharmacology and Physiology, 22, 276-286.

Ghafourian, T., Samaros, E., Brooks, J. \& Riviere, J.E. (2010) Validated models for predicting skin penetration from different vehicles. European Journal of Pharmaceutical Science, 41, 612-616.

Herd, R.P., Sams, R.A. \& Ashcraft, S.M. (1996) Persistence of ivermectin in plasma and faeces following treatment of cows with ivermectin sustained-release, pour-on or injectable formulations. International Journal of Parasitology, 26, 1087-1093.

Herkenne, C., Alberti, I., Naik, A., Kalin, Y.N., Mathy, F.X., Preat, V. \& Guy, R.H. (2008) In Vivo methods for the assessment of topical drug bioavailability. Pharmaceutical Research, 25, 87-103.

Higuchi, I. (1961) Rate of release of medicaments from ointment bases containing drugs in suspension. Journal of Pharmaceutical Science, 50, 874-875.

ICH Harmonised Tripartite Guideline (2005) 'Validation of analytical procedures: text and methodology Q2(R1)' Current Step 4 version; Parent Guideline dated 27 October 1994 (complementary guideline on methodology dated 6 November 1996 incorporated in November 2005).

Jenkinson, D.M., Hutchison, G., Jackson, D. \& McQueen, L. (1986) Route of passage of cypermethrin across the surface of sheep skin. Research in Veterinary Science, 41, 237-241.

Kyles, A.E., Papich, M. \& Hardie, E.M. (1996) Disposition of transdermally administered fentanyl in dogs. American Journal of Veterinary Research, 57, 715-719.

Laffont, C.M., Alvinerie, M., Bousquet-Mélou, A. \& Toutain, P.L. (2001) Licking behaviour and environmental contamination arising from pour-on ivermectin for cattle. International Journal of Parasitology, 31, 1687-1692.

Lee, D.D., Papich, M.G. \& Hardie, E.M. (2000) Comparison of pharmacokinetics of fentanyl after intravenous and transdermal administration in cats. American Journal of Veterinary Research, 61, 672-677.

Lehman, P.A., Raney, S.G. \& Franz, T.J. (2011) Percutaneous absorption in man: in vitro - in vivo correlation. Skin Pharmacology and Physiology, 24, 224-230.

Martinez, M., Langston, C., Martin, T. \& Conner, D. (2002) Challenges associated with the evalution of veterinary product bioequivalence: an AAVPT perspective. Journal of Veterinary Pharmacology and Therapeutics, 25, 201-220.

Mills, P.C. \& Cross, S.E. (2006) Transdermal drug delivery: basic principles for the veterinarian. Veterinary Journal, 172, 218-233.

Monteiro-Riviere, N.A. (1991) Comparative anatomy, physiology, and biochemistry of mammalian skin. In Dermal and Ocular Toxicology: 
Fundamentals and Methods. Ed. Hobson, D.W., pp. 3-71. CRC Press Inc., Florida.

Monteiro-Riviere, N.A. (2006) The integument. In Dellman's Textbook of Veterinary Histology, 6th edn. Eds Eurell, J.A. \& Frappier, B.L., pp. 320349. Blackwell Publishing, Iowa.

Monteiro-Riviere, N.A. (2008a) Anatomical factors that affect barrier function. In Dermatotoxicology, 7th edn. Eds Zhai, H., Wilhelm, K.P. \& Maibach, H.I., pp. 39-50. CRC Press, Florida.

Monteiro-Riviere, N.A., Bristol, D.G., Manning, T.O., Rogers, R.A. \& Riviere, J.E. (1990) Interspecies and interregional analysis of the comparative histologic thickness and laser Doppler blood flow measurements at five cutaneous sites in nine species. Journal of Investigative Dermatology, 95, 582-586.

Monteiro-Riviere, N.A., Inman, A.O., Riviere, J.E., McNeill, S.C. \& Francoeur, M.L. (1993) Topical penetration of piroxicam is dependent on the distribution of the local cutaneous vasculature. Pharmaceutical Research, 10, 1326-1331.

Monteiro-Riviere, N.A., Baynes, R.E. \& Riviere, J.E. (2008b) Animal skin morphology and dermal absorption. In Dermal Absorption and Toxicity Assessment, 2nd edn. Eds Roberts, M.S. \& Walters, K.A., pp. 17-35. Informa Healthcare, New York.

Muhammad, F., Brooks, J.D. \& Riviere, J.E. (2004) Comparative mixture effects of JP-8 (100) additives on the dermal absorption and disposition of jet fuel hydrocarbons in different membrane model systems. Toxicology Letters, 150, 351-365.

Narkar, Y. (2010) Bioequivalence for topical products - an update. Pharmaceutical Research, 27, 2590-2601.

N’Dri-Stempfer, B., Navidi, W.C., Guy, R.H. \& Bunge, A.L. (2009) Improved bioequivalence assessment of topical dermatological drug products using dermatopharmacokinetics. Pharmaceutical Research, 26, 316-328.

OECD (2004) Guidance document for the conduct of skin absorption studies. OECD Environmental Health and Safety Publication. Series on Testing and Assessment No. 28, Paris: Environment Directorate, Organization for Economic Co-operation and Development, March, 2004.

Reifenrath, W.G., Ross, J.H. \& Driver, J.H. (2011) Experimental methods for determining permethrin dermal absorption. Journal of Toxicology and Environmental Health A, 74, 325-335.
Riviere, J.E. \& Brooks, J.D. (2005) Predicting skin permeability from complex chemical mixtures. Toxicology and Applied. Pharmacology, 208, 99-110.

Riviere, J.E. \& Brooks, J.D. (2011) Predicting skin permeability from complex chemical mixtures: dependency of quantitative structure permeability relationships (QPSR) on biology of skin model used. Toxicological. Sciences, 119, 224-232.

Riviere, J.E., Baynes, R.E., Brooks, J.D., Yeatts, J.L. \& Monteiro-Riviere, N.A. (2003) Percutaneous absorption of topical diethyl-m-toluamide (DEET): effects of exposure variables and coadministered toxicants. Journal of Toxicology and Environmental Health. A, 66, 133-151.

Riviere, J.E., Baynes, R.E. \& Xia, X.R. (2007) Membrane coated fiber (MCF) array approach for predicting skin permeability of chemical mixtures from different vehicles. Toxicological Sciences, 99, 153-161.

Riviere, J.E., Brooks, J.D., Yeatts, J.L. \& Koivisto, E. (2010) Surfactant effects on skin absorption of model organic chemicals: implications for dermal risk assessment studies. Journal of Toxicology and Environmental Health. Part A, 73, 725-737.

Ross, J.H., Reifenrath, W.G. \& Driver, J.H. (2011) Estimation of the percutaneous absorption of permethrin in humans using the parallelogram method. Journal of Toxicology and Environmental Health A, 74, 351-363.

Sarasola, P., Jernigan, A.D., Walker, D.K., Castledine, J., Smith, D.G. \& Rowan, T.G. (2002) Pharmacokinetics of selamectin following intravenous, oral and topical administration in cats and dogs. Journal of Veterinary Pharmacology and Therapeutics, 25, 265-272.

Shah, V.P., Elkins, J., Hanus, J., Noorizadeh, C. \& Skelley, J.P. (1991) In vitro release of hydrocortisone from topical preparations and automated procedure. Pharmaceutical Research, 8, 55-59.

Skelley, J.P., Shah, V.P., Maibach, H.I., Guy, R.H. \& Wester, R.C. (1987) FDA and AAPS report of the workshop on principles and practices of in vitro percutaneous penetration studies: relevance to bioavailability and bioequivalence. Pharmaceutical Research, 4, 265-267.

Toutain, P.L., Modric, S., Bousquet-Melou, A., Sallovitz, J.M. \& Lanusse, C. (2012) Licking behavior be considered in the bioavailability evaluation of transdermal products?. Journal of Veterinary Pharmacology and Therapeutics, 35 (Suppl. 1), 25-29.

WHO (World Health Organization) (2005) International programme on chemical safety, dermal absorption. Environmental Health Criteria 235. WHO Press, Geneva, Switzerland. 